\title{
ABSENTEÍSMO NAENFERMAGEM EM UTI NEONATAL: PERFIL DO PROFISSIONAL E MOTIVOS DAS AUSÊNCIAS
}

\author{
Ilana de Castro ${ }^{1}$, Elizabeth Bernardino² ${ }^{2}$ Elaine Lorena Zen Ribeiro ${ }^{3}$
}

\begin{abstract}
RESUMO: Estudo quantiqualitativo do tipo descritivo, realizado nos meses de agosto a setembro de 2006 em uma UTI neonatal de Curitiba. Os objetivos foram descrever o perfil dos trabalhadores implicados no absenteísmo desta unidade e identificar os motivos das ausências não previstas desses trabalhadores. A amostra foi constituída de 23 profissionais que somaram 34 afastamentos. Foi aplicado um questionário com perguntas abertas e fechadas. Os dados quantitativos foram apresentados em forma de tabelas e os qualitativos foram categorizados. Os resultados mostraram que $73 \%$ eram Auxiliares de Enfermagem com idade entre 31 a 40 anos (47\%), casada (68\%), com filhos (68\%) e com um emprego (76\%). Os motivos mais observados foram licença para cuidar da própria saúde (44\%) e para cuidar de familiar (32\%). Sugere-se que se estude qual o impacto destas ausências, tanto na saúde dos trabalhadores como na assistência prestada.
\end{abstract}

PALAVRAS-CHAVE: Absenteísmo; Equipe de enfermagem; Unidades de terapia intensiva neonatal.

\section{NURSING ABSENTEEISM AT NEONATAL ICU: PROFESSIONALS' PROFILE AND ABSENCE REASONS}

\begin{abstract}
Quali-quantitative study of descriptive type carried out at a neonatal ICU in Curitiba /Brazil from August to September/2006. It aimed to describe the profile of the professionals implicated in the absenteeism in this unit and to identify the reasons for their unpredictable absences. The sample entailed 23 professionals who added up to 34 absences. A questionnaire with open and closed questions was applied. Quantitative data were displayed in table form and qualitative ones were categorized. Results showed that 73\% were nursing assistants, ages ranging from 31 and 40 (47\%), married (68\%), having children (68\%), having a job (76\%). The reasons observed were sick leave requested on behalf of their own health (44\%) or of a family member's (32\%). It is suggested that the impact of those absences be studied, not only on professionals' health but also on care delivery.
\end{abstract}

KEYWORS: Absenteism; Nursing team; Neonatal intensive care units.

\section{ESTUDIO SOBRE LAS CAUSAS DELABSENTISMO EN LA ENFERMERÍA EN UCI NEONATAL}

RESUMEN: Estudio cuantitativo e cualitativo del tipo descriptivo, realizado durante los meses de agosto y septiembre de 2006 en una CTI para recién nacidos de Curitiba. Tiene como objetivos describir el perfil de los trabajadores implicados en el absentismo de esta unidad e identificar los motivos de ausencia imprevistas de estos trabajadores. La muestra fue formada por 23 profesionales que sumaron 34 ausencias. Fue aplicado un cuestionario con preguntas abiertas y cerradas. Los datos cuantitativos fueron presentados en forma de tablas y los cualitativos fueron clasificados por categorías. Los resultados mostraron que 73\% eran auxiliares de enfermería con edades entre 31 y 40 años (47\%), casados (68\%), con hijos (68\%), y con un empleo (76\%). Los motivos más observados fueron licencia por salud (44\%) y para cuidad de familiar (32\%). Se sugiere el estudio del impacto de esas ausencias, tanto en la salud de los trabajadores como en la asistencia prestada.

PALABRAS CLAVE: Absentismo; Grupo de enfermería; Unidades de terapia intensiva neonatal.

${ }^{1}$ Graduanda do Curso de Graduação em Enfermagem da Universidade Tuiuti do Paraná.

${ }^{2}$ Enfermeira. Doutora em Enfermagem pela EEUSP. Professora Adjunta da Universidade Tuiuti do Paraná.

${ }^{3}$ Enfermeira. Chefe de Enfermagem da Unidade de Terapia Intensiva Neonatal do Hospital de Clínicas.

Autor correspondente:

Elaine Lorena Zen Ribeiro

Rua Professora Etelvina Durslli, 800 - 80660-000 - Curitiba-PR

Recebido: 02/05/07

E-mail: eldinezen@ufpr.br

Aprovado: 09/07/08

Cogitare Enferm 2008 Jul/Set; 13(3):374-9 


\section{INTRODUÇÃO}

Absenteísmo pode ser definido como as ausências nos momentos em que os empregados deveriam estar trabalhando normalmente ${ }^{(1)}$. Esse conceito pode incluir até os atrasos, pois se refere ao somatório dos períodos em que os profissionais, de determinada organização, se ausentam do trabalho. Deste modo, o absenteísmo pode ser entendido de diversas maneiras haja vista que também se refere às ausências de trabalho devido a licenças, férias, faltas ${ }^{(2)}$ e inclui as suspensões ${ }^{(3)}$ ou as ausências por faltas e licenças médicas, denominadas ausências não programadas ou não previstas, porém exclui férias e folgas porque são consideradas ausências planejadas dentro de uma jornada de trabalho legal ${ }^{(4)}$.

Na enfermagem, o absenteísmo merece especial atenção, sobretudo nos serviços de funcionamento ininterrupto, pelas implicações na redução da equipe e na assistência prestada aos pacientes ${ }^{(5)}$. A definição mais utilizada, na enfermagem, entende absenteísmo como as ausências previstas relativas às folgas e férias e as ausências não previstas referentes às faltas, licenças e suspensões ${ }^{(3)}$. As ausências não previstas referem-se à soma de uma série de ausências como faltas e todo tipo de licença médica; como por exemplo, licença maternidade, licença-prêmio, por acidente de trabalho, de saúde, gala e nojo ${ }^{(5)}$.

Por ter suas causas ligadas a diversos fatores, o absenteísmo se torna complexo e de difícil gerenciamento $^{(6)}$. Sua etiologia pode estar relacionada às condições de trabalho encontradas nas empresas, como, estilo de liderança e controle, repetitividade das tarefas e também a falta de integração entre funcionários, que interfere indiretamente na assiduidade do trabalhador ${ }^{(7)}$.

Ressalta-se além de causas relacionadas à organização do trabalho, a relação existente entre o sexo feminino e o absenteísmo, uma vez que o número de mulheres continua a crescer no mercado de trabalho e é predominante na área de Enfermagem. Neste sentido, a dupla atividade no trabalho e no lar pode implicar sobrecarga física, preocupações e pouco repouso, desencadeando diversas doenças, que podem resultar em absenteísmo ${ }^{(8)}$. A profissional de Enfermagem desenvolve seu trabalho remunerado e, ao mesmo tempo, gerencia sua vida como pessoa, esposa e mãe, ${ }^{(9)}$ um somatório de atribuições realizadas que contribui, muitas vezes, para seu desgaste físico e emocional. A remuneração das profissionais de
Enfermagem também pode estar associada ao absenteísmo, pois os salários baixos podem implicar na necessidade de dois ou até mais empregos causando sobrecarga, que pode levar ao absenteísmo.

O absenteísmo é considerado um problema administrativo para qualquer organização, sendo ainda mais grave nas instituições de saúde, pois além de gerar custo/prejuízo para a instituição, pode interferir na produtividade dos trabalhadores, comprometer a qualidade da assistência e, conseqüentemente afetar diretamente a recuperação do cliente. Além disso, o absenteísmo se torna ainda mais visível e problemático nos ambientes críticos, como é o caso de Unidades de Terapia Intensiva, Prontos Socorros e também Centros Cirúrgicos, cujos cuidados e recursos tecnológicos requerem mão-de-obra qualificada, dificilmente substituível.

Durante a realização do estágio de Administração em Enfermagem em uma UTI neonatal da cidade de Curitiba, foi percebido e relatado, pelas enfermeiras do setor, a dificuldade em manter uma escala de trabalho devido às ausências constantes das funcionárias do serviço de enfermagem, ocasionando transtornos durante a jornada de trabalho.

Na UTI Neonatal, o absenteísmo pode acentuar os problemas já existentes, uma vez que a população atendida nessa unidade são recém-natos instáveis do ponto de vista clínico, com diferentes patologias e prognósticos, cujos cuidados são realizados por profissionais que detêm determinadas competências e habilidades para lidar, tanto com o aporte tecnológico como com as especificidades destes pacientes. Com estas características, a ausência do funcionário pode resultar no não atendimento da necessidade do paciente, gerar sobrecarga/desgaste dos profissionais de Enfermagem e incidir em implicações de ordem legal para a instituição. Diante dessa problemática objetivouse: descrever o perfil dos trabalhadores implicados no absenteísmo desta unidade e identificar os motivos das ausências não previstas desses trabalhadores.

\section{METODOLOGIA}

Trata-se de um estudo quali-quantitativo, do tipo descritivo cujo cenário foi uma UTI Neonatal de um hospital público, localizado em Curitiba. O serviço possui 24 leitos, os quais são destinados a internação de recém-nascidos portadores das mais diversas patologias, ou seja, com um perfil de morbidade heterogêneo. A equipe de Enfermagem é constituída 
por 67 profissionais do sexo feminino: 10 enfermeiras, 46 auxiliares e 11 técnicas em Enfermagem, distribuídas em três turnos de trabalho.

A amostra foi intencional e a coleta de dados aconteceu nos meses de agosto a setembro de 2006, pela aplicação de um questionário com 15 perguntas abertas e fechadas. Para descrever o perfil foram utilizadas as variáveis: categoria profissional, faixa etária, estado civil, número de filhos e número de empregos, pressupondo que estas teriam impacto no absenteísmo. Os motivos foram apreendidos tanto de uma questão fechada como da análise dos discursos das questões abertas. Os dados quantitativos foram apresentados sob forma de tabelas e os dados qualitativos foram categorizados. Ambos foram analisados à luz da literatura, utilizando referenciais relacionados ao absenteísmo.

A coleta de dados se deu da seguinte maneira: todos os dias, em todos os turnos, identificava-se, pela escala de trabalho, as ausências não previstas. Em seguida, procurava-se a funcionária faltosa e explicavase a pesquisa e, quando esta aceitava participar, era oferecido o questionário, de 15 questões, das quais 12 eram respondidas por ela e três por sua chefia. As questões que a chefe deveria responder eram: a funcionária avisou que iria faltar, se a chefia conseguiu repor a falta e se a funcionária apresentou algum documento comprobatório; como atestado.

Durante este período foram registradas 48 ausências não previstas, ou seja, com faltas por qualquer outro motivo que não seja férias, folga ou licença maternidade. Destas, 19\% das funcionárias se recusaram a participar da pesquisa por motivos diversos e $10 \%$ permaneceram afastadas do serviço durante toda a coleta de dados, o que impediu sua participação. Os sujeitos da pesquisa foram, portanto, 23 profissionais, que durante estes dois meses, tiveram ausências e que aceitaram participar voluntariamente da pesquisa. Juntas, somaram 34 afastamentos (102 dias), pois 26\% delas tiveram mais de uma ausência.

A pesquisa foi aprovada pelo Comitê de Ética em Pesquisa com o número CEP/HC: 1255.103/200607, e obedeceu a Resolução 196/96, ${ }^{(10)}$ no que concerne ao sigilo das informações, confiabilidade dos dados, garantia de anonimato e divulgação dos resultados. Todos os sujeitos da pesquisa foram devidamente informados sobre os riscos e benefícios e assinaram o Termo de Consentimento Livre e Esclarecido.

\section{RESULTADOS E DISCUSSÃO}

\section{Perfil das profissionais de enfermagem implicadas nas ausências}

Os 23 profissionais da Equipe de Enfermagem totalizaram 34 ausências não previstas durante o período do estudo. A categoria que apresentou maior número de ausências foi a dos Auxiliares de Enfermagem (25\%), seguida dos Enfermeiros (15\%) e dos Técnicos em Enfermagem (12\%). Este resultado é esperado, uma vez que a categoria dos Auxiliares constitui o maior número de sujeitos da pesquisa, explicitando, também, que compõem a maioria das 67 pessoas desta equipe, trabalhando atualmente, nesta UTI. Sobre este tema, tem-se o estudo ${ }^{(11)}$ no qual os Auxiliares de Enfermagem apresentaram $72 \%$ de afastamentos e outro $^{(12)}$ com um número maior, $77 \%$ de ausências.

Tabela 1 - Faixa etária e número de afastamentos. Curitiba, 2006

\begin{tabular}{ccc}
\hline Idade & $\begin{array}{c}\text { Número de } \\
\text { afastamentos }\end{array}$ & $\%$ \\
\hline $21-30$ anos & 07 & 21 \\
$31-40$ anos & 16 & 47 \\
$41-50$ anos & 09 & 26 \\
$51-60$ anos & 02 & 06 \\
\hline Total & 34 & 100 \\
\hline
\end{tabular}

A maioria das funcionárias com ausências não previstas (68\%), possui entre 21 e 40 anos de idade e se caracteriza como uma faixa etária jovem. Um outro estudo $^{(13)}$ apresentou que 65\% das Enfermeiras e 54\% das Auxiliares de Enfermagem estão na faixa etária entre 30 a 40 anos, caracterizando, também, um grupo de profissionais jovens. Os outros $26 \%$ são considerados adultos jovens e, somente 6\% estão na faixa dos 51-60 anos os quais podem ter suas ausências associadas ao desgaste fisiológico natural resultante da idade.

Tabela 2 - Estado civil dos respondentes e número de profissionais, Curitiba, 2006

\begin{tabular}{ccc}
\hline Estado civil & $\begin{array}{c}\text { Número de } \\
\text { profissionais }\end{array}$ & $\%$ \\
\hline Solteira & 06 & 17 \\
Casada & 23 & 68 \\
Divorciada & 04 & 12 \\
Viúva & 01 & 03 \\
\hline Total & 34 & 100 \\
\hline
\end{tabular}


Os dados apontam que a maioria (68\%) das profissionais é casada, o que pode ou não interferir no aumento do absenteísmo. Este dado é importante porque a mulher executa atividades laborais e domésticas em uma associação de tarefas que pode resultar em cansaço e estresse, pois geralmente consegue compartilhar muito pouco de suas obrigações com o companheiro ${ }^{(9)}$. No entanto, há poucos estudos que atribuem esta mesma sobrecarga às solteiras, viúvas e divorciadas, mesmo sabendo que também possuem responsabilidades ditas “domésticas”.

Tabela 3 - Distribuição do número de filhos dos sujeitos da pesquisa. Curitiba, 2006

\begin{tabular}{ccc}
\hline Filho & $\begin{array}{c}\text { Número de } \\
\text { profissionais }\end{array}$ & $\%$ \\
\hline Nenhum & 11 & 32 \\
1 & 06 & 18 \\
2 & 11 & 32 \\
$\geq 3$ & 06 & 18 \\
\hline Total & 34 & 100 \\
\hline
\end{tabular}

A variável número de filhos mostra que 32\% das profissionais não possuem filhos, mas $68 \%$ os possuem. O número de filhos pode ser um complicador na medida em que, além das atividades profissionais, a mulher é responsável, na grande maioria das famílias, pela guarda, pelo cuidado e também pela educação dos filhos, resultando em acúmulo de atividades ${ }^{(9)}$. A Enfermagem é uma profissão essencialmente feminina, sendo elevado o número de pessoas que vivencia essa realidade diariamente de serem mães e ao mesmo tempo profissionais, com toda complexidade que a referida situação comporta. A dificuldade para conciliar essas atividades foi relatada por algumas profissionais:

Não é fácil cuidar dos filhos e parentes dos outros deixando seus próprios filhos em casa, chorando e te chamando (E9).

Com relação à variável número de empregos, observou-se que $76 \%$ possuem um emprego; $18 \%$ dois e $6 \%$ três ou mais. Acreditava-se, no início do estudo, que o número de empregos teria maior impacto nas ausências o que não se confirmou, pois $76 \%$ das profissionais possui somente um vínculo empregatício.

Motivos das ausências, número de dias e a substituição dos profissionais
As tabelas apresentadas a seguir, descrevem os motivos das ausências não previstas, o período de afastamento e a taxa de substituição das profissionais implicadas no absenteísmo.

Tabela 4 - Motivos de ausências não previstas e número de vezes que estes aparecem. Curitiba, 2006

\begin{tabular}{ccc}
\hline $\begin{array}{c}\text { Motivo de } \\
\text { ausências não } \\
\text { previstas }\end{array}$ & $\begin{array}{c}\text { Número de } \\
\text { ausências não } \\
\text { previstas }\end{array}$ & $\%$ \\
\hline $\begin{array}{c}\text { Licença de saúde } \\
\text { Licença de }\end{array}$ & 15 & 44 \\
saúde/familiar & 11 & 32 \\
Motivo particular & 05 & 15 \\
Licença luto & 02 & 06 \\
Licença congresso & 01 & 03 \\
\hline Total & 34 & 100 \\
\hline
\end{tabular}

Os motivos declarados pelas trabalhadoras mostram que a licença para cuidar da própria saúde (44\%) é o maior motivo de ausência, seguido pela licença para cuidar de familiar (32\%), motivos particulares (15\%), luto (6\%) e estudo (3\%).

O percentual de $44 \%$, que representa as ausências ocasionadas por problemas de saúde do próprio trabalhador, confirma os achados em outros estudos. Nestes, os percentuais podem apresentar grandes variações como de $46 \%,{ }^{(6)}, 75 \%,{ }^{(8)}, 79 \%{ }^{(1)} \mathrm{e}$, até $88 \%{ }^{(14)}$.

Das licenças de saúde, 68\% apresentaram atestados médicos como justificativa da ausência. $\mathrm{O}$ atestado médico é, muitas vezes, um escape que o trabalhador encontra para fugir de situações indesejáveis, conflituosas e até mesmo para demonstrar insatisfação ${ }^{(7)}$.

A licença de saúde/familiar também é responsável por 32\% dos afastamentos ressaltandose que ao averiguar o problema de saúde apresentado na família, 8 destes afastamentos está relacionado a doença dos filhos.

As 34 ausências do período dos meses agosto e setembro de 2006 somaram 102 dias de afastamento e, a maioria (79\%) foi de 1 a 5 dias. Resultado semelhante foi obtido em outro estudo sobre o absenteísmo, no qual a maioria dos funcionários apresentou período de afastamento de 1 a 4 dias $^{(14)}$.

Atenta-se para o fato de que, quanto maior o período de afastamento, maior será o número de profissionais necessárias para substituição, podendo 
acarretar prejuízos na assistência bem como sobrecarga de trabalho e desgaste das demais integrantes da equipe.

O estudo também permitiu verificar que $62 \%$ das ausências não foram substituídas, fato que pode ser explicado parcialmente porque $56 \%$ das funcionárias comunicou seu afastamento em um período menor ou igual a 5 horas, dificultando a reposição. Pode-se inferir, portanto, que quanto menor o período para a comunicação da ausência, menor também é a taxa de substituição devido às dificuldades de encontrar substitutos em tempo hábil.

Somente $21 \%$ das funcionárias relacionaram a ausência com o tipo e/ou as condições de trabalho, número que deve ser considerado, uma vez que os motivos associados foram stress, rotinas do serviço, sobrecarga física e emocional.

Alguns estudos ${ }^{(7,14)}$ afirmaram que o número insuficiente de recursos humanos, condições inadequadas de trabalho, divisão de tarefas, rígida hierarquização para o cumprimento de rotinas, normas e regulamentos também contribuem para o aumento do índice de absenteísmo e podem acarretar em agravos à saúde, de natureza física ou psicológica.

A UTI é geradora de estresse por ser uma unidade fechada, pela natureza do cuidado prestado com pacientes que necessitam de monitoramento constante e por ter muitos equipamentos e ruídos ${ }^{(15)}$. Tais dificuldades foram apreendidas nas falas a seguir:

Estresse associado à rotina diária. Sobrecarga de trabalho (E.3).

Trabalho muito, estresse, uma doença atrás da outra (E.9).

Cansaço físico e emocional, estamos trabalhando no limite, assumindo vários pacientes e continuam internando. Não existe respeito com a equipe de Enfermagem (E.12).

As falas sugerem o cansaço provocado pelas condições de trabalho. As características do cuidado realizado, o déficit de profissionais, a falta de reposição e a reposição por horas extras realizadas pelos próprios profissionais, parecem centrais nas possíveis explicações para as ausências observadas na unidade.

\section{CONSIDERAÇÕES FINAIS}

A investigação constatou que 23 dos 67 profissionais da UTI neonatal somaram 34 ausências não previstas. A maioria (47\%) das profissionais com ausências pertence a uma faixa etária jovem com idade entre 31 a 40 anos, é casada (68\%) possui filhos (68\%) e tem somente um emprego (76\%). A categoria Auxiliar de Enfermagem foi responsável pelo maior número de afastamentos, totalizando 73\%. Dentre os motivos das ausências, houve predomínio de doença do próprio trabalhador (44\%), seguida de licença para tratamento/ doença de familiares (32\%), motivos particulares (15\%), licença luto (6\%) e congresso (3\%).

Com a realização deste estudo apreende-se a importância de conhecer o absenteísmo na UTI Neonatal e de aprofundar o conhecimento acerca desta temática. Ainda, aponta para discussões sobre aspectos voltados a adequação quantitativa de recursos humanos, condições laborais e de (re)organização do trabalho nesta Unidade.

Como sugestão, a unidade poderia considerar a possibilidade de monitorar sua taxa de absenteísmo. Para obtê-la é preciso ter registros precisos e controle efetivo das ausências. Além de um aprofundamento da origem das doenças que estão afastando suas funcionárias, sugere-se ainda, que se estude qual o impacto destas ausências na assistência, monitorando as iatrogenias causadas especificamente pela equipe de enfermagem.

\section{REFERÊNCIAS}

1. Echer IC, Moura GM, Magalhães AM, Piovesan R. Estudo do absenteísmo como variável no planejamento de recursos humanos em enfermagem. Rev Gaúcha Enferm. 1999 Jul;20(2):65-76.

2. Duarte ERM, Magalhães AMM, Moura GMSS. Estudo das variáveis que participam do dimensionamento do pessoal de enfermagem em hospitais de grande porte. Rev Gaúcha Enferm. 1995;16(1/2):5-16.

3. Gaidizinski RR, Sanna MC, Leite MMJ, Mayor ERC. Estudo das ausências da equipe de enfermagem num hospital geral de grande porte. Rev Enferm Complexo HC/FMUSP. 1998;1(4):8-14.

4. Jorge, AL. Motivos que levam os trabalhadores de enfermagem ao absenteísmo. Acta Paul Enferm. 1995;8(1):39-46.

5. Gaidzinski RR, Fugulin FMT, Castilho V. Dimensionamento de pessoal de enfermagem em instuições de saúde. In: Kurcgant P, coordenador. 
Gerenciamento de enfermagem. Rio de Janeiro: Guanabara Koogan; 2005.

6. Nascimento GM. Estudo do absenteísmo dos trabalhadores de enfermagem em uma unidade básica e distrital de saúde do município de Ribeirão Preto - SP [dissertação]. Ribeirão Preto (SP): Universidade de São Paulo; 2003.

7. Alves M. As causas do absenteísmo na enfermagem: uma dimensão do sofrimento no trabalho [tese]. São Paulo (SP): Universidade de São Paulo; 1996.

8. Azevedo CAB, Nogueira DP. Absenteísmo - doença em mulheres. Rev Bras Saúde Ocupac. 1982 Abr/ Jun;38(10):48-51.

9. Santos RS, Spindola T. Mulher e trabalho - a história de vida de mães trabalhadoras em enfermagem. Rev LatinoAm Enferm. 2003 Set/Out;11(5):593-600.

10. Ministério da Saúde (BR). Conselho Nacional de saúde. Resolução 196/96. Diretrizes e normas regulamentadoras de pesquisas envolvendo seres humanos. Disponível em: www.datasus.gov.br/conselho/comissoes/etica/ conep.htm. Acesso em: 12 junho 2006.

11. Delgado LM, Oliveira BRG. Perfil epidemiológico do adoecimento dos profissionais de enfermagem de um hospital universitário. Rev Nursing. 2005 Ago;87(8):365-70.

12. Marziale MHP, Silva DMPP. Problemas de saúde responsáveis pelo absenteísmo de trabalhadores de enfermagem de um hospital universitário. Acta Scientiarum. 2003 Jan/Jun; 5(1):191-7.

13. Beck CLC, Donaduzzi JC, Gonzales RMB, Stekel LMC. $\mathrm{O}$ trabalho da enfermagem em unidades críticas e sua repercussão sobre a saúde dos trabalhadores. Esc Anna Nery Rev Enferm. 2006 Ago;10(4):221-7.

14. Barboza DB, Soler ZASG. Afastamentos do trabalho na enfermagem: ocorrências com trabalhadores de um hospital de ensino. Rev Latino-Am Enferm. 2003 Mar/ Abr;11(2):177-83.

15. Costenaro RGS, Medeiros HMF, Santini AM, Zaberlan C. Estresse: vivência profissional de enfermeiras que atuam em UTI neonatal. Cogitare Enferm. 2005 Set/ Dez;10(3):14-21. 